\title{
Primary Biliary Cirrhosis in a Male: A Case Report and Review of Literature
}

\author{
SAMI RA RAHAT AFROZE, ${ }^{1}$ MUHAMMAD ABDUR RAHI M, ${ }^{2}$ MD. MEHEDI HASAN, ${ }^{3}$ FARZANA AFROZ, ${ }^{1}$ \\ HASNA FAHMI MA HAQUE, ${ }^{1} \mathrm{~J}$ AMAL UDDI N AHMED, ${ }^{4}$ AKM SHAHEEN AHMED, ${ }^{5}$ MD. DELWAR HOSSAI N ${ }^{5}$ \\ MD. RAZIUR RAHMAN, ${ }^{5}$ AKM MUSA, ${ }^{6}$ KHWAJ A NAZI M UDDI N ${ }^{6}$
}

\begin{abstract}
A middle aged woman presenting with fatigue, pruritus, xanthelesma and hepatomegaly remains the typical presentation of primary biliary cirrhosis. Here, we present a case of primary biliary cirrhosis occurring in a male patient, who presented with fatigue and pruritus, had hepato-splenomegaly, raised serum alkaline phosphatase, positive anti-mitrochondrial antibody and negative antinuclear antibody.
\end{abstract}

Key words: anti-mitrochondrial antibody, antinuclear antibody, male, primary biliary cirrhosis.

\section{Introduction}

Primary biliary cirrhosis (PBC) is a chronic and slowly progressive cholestatic liver disease of autoimmune etiology characterized by granulomatous inflammation of the intrahepatic bile ducts. A continuous assault on the bile duct epithelial cells lead to their gradual destruction and eventual disappearance. The sustained loss of intralobular bile ducts causes the signs and symptoms of cholestasis and eventually results in cirrhosis and liver failure. ${ }^{1}$ The onset is usually between the ages of 30 to 65 years $^{2}$ with a 9:1 female-to-male ratio. ${ }^{3}$ According to the American Association for the Study of Liver Diseases (AASLD) the diagnosis is established when two of the following three criteria are met: ${ }^{2}$

a) Biochemical evidence of cholestasis based mainly on alkaline phosphatase elevation

b) Presence of antimitrochondrial antibody (AMA)

c) Histologic evidence of non-suppurative destructive cholangitis and destruction of interlobular bile ducts.

1. Registrar, Dept. of Internal Medicine, BIRDEM, Dhaka.

2. Assistant Professor, Dept. of Nephrology, BIRDEM, Dhaka.

3. Junior Consultant, Dept. of Surgery, Kurmitola General Hospital, Dhaka.

4. Assistant Professor, Dept. of Internal Medicine, BIRDEM, Dhaka.

5. Associate Professor, Dept. of Internal Medicine, BIRDEM, Dhaka.

6. Professor, Dept. of Internal Medicine, BIRDEM, Dhaka.

Correspondence: Dr. Samira Rahat Afroze, FCPS (Medicine), Registrar, Department of Internal Medicine, BIRDEM, Dhaka. Bangladesh. Email: sra@dhaka.net.

\section{Case report}

A 52-year-old diabetic, smoker (15 pack year) presented with a six month history of fatigue and weight loss of six kg. For the last two weeks he developed generalized pruritus which did not respond to anti-histamines. He did not have any fever, respiratory, cardiac, joint symptoms, past history of tuberculosis (TB) or TB contacts. His appetite was normal. Prior to admission he was diagnosed as Hashimoto's thyroiditis on the basis of thyroid function tests (TSH- 17.80 $\mu \mathrm{IU}$, FT4- $8.1 \mathrm{pmol} / \mathrm{L}$, FT3-1.2 pmol/L), positive antithyroid antibodies and was on thyroxin replacement (50 $\mu \mathrm{gm}$ daily).

He was anaemic, there was no lymphadenopathy, jaundice or other stigmata of chronic liver disease. Scratch marks all over his body and shiny nails were present. He had a firm, non-tender, smooth-surfaced hepatomegaly $(5.5 \mathrm{~cm})$ and a just palpable spleen without ascites. Other systemic examination findings were normal.

Investigations showed thrombocytopenia $(35,000 / \mathrm{cmm})$, eosinophilia (14\%), raised serum alkaline phosphatase (196 IU/L), mild elevation of aspartate aminotransferase (66 IU/L) and alanine aminotransferase (48 IU/L). Serum albumin was $30 \mathrm{~g} / \mathrm{L}$ and bilirubin \& prothrombin time was normal. Ultrasonogram of abdomen revealed mild hepato-splenomegaly. An upper GIT endoscopy revealed Grade I esophageal varices with portal hypertensive gastropathy. Magnetic resonance cholangiopancreatogram (MRCP) excluded other intrahepatic (Fig 1) and extrahepatic causes of cholestasis (Fig 2). Fibroscan of his liver revealed severe liver stiffness (69.1 KPa, Fig 3). 


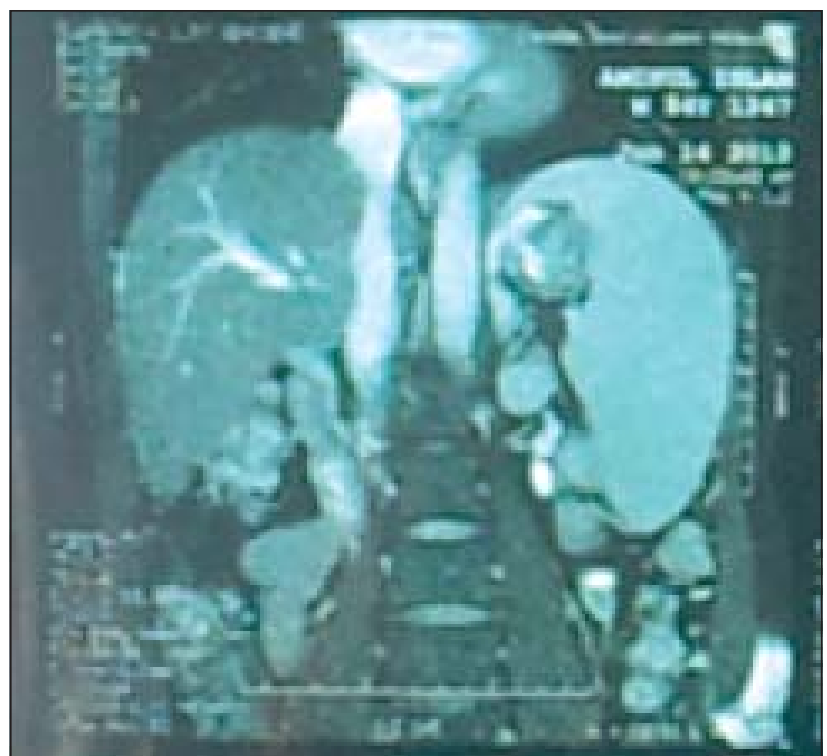

Fig.-1: MRCP showing hepatosplenomegaly with normal intrahepatic biliary channels.

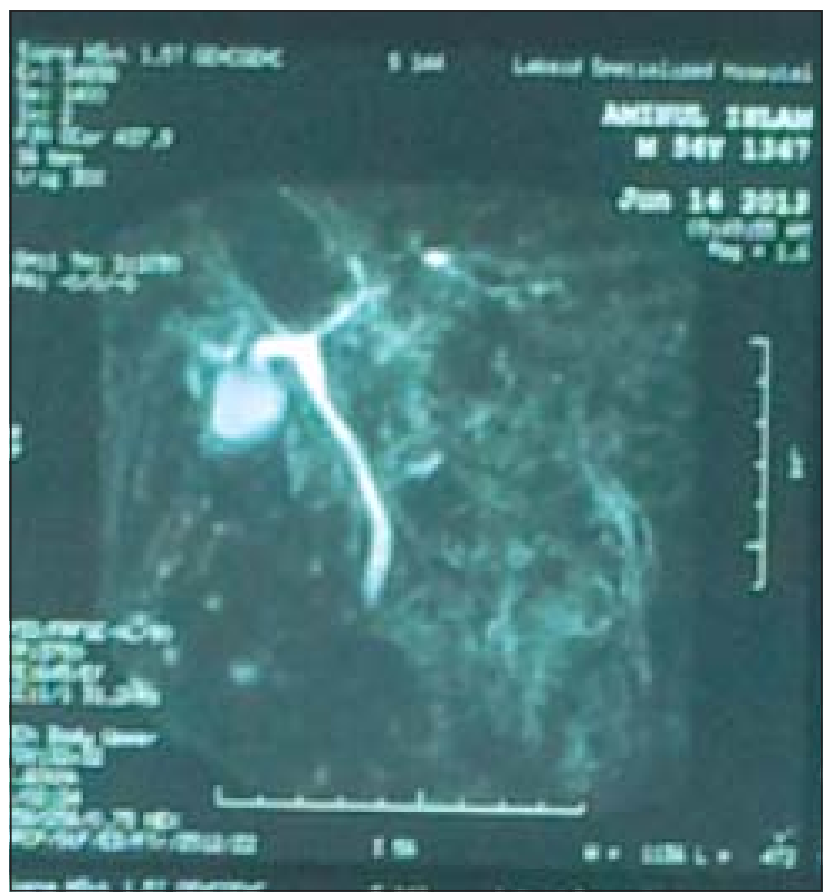

Fig.-2: MRCP showing normal extrahepatic biliary channels

Antimitrochondrial antibody (AMA) was positive (10.1units, reference value $>1.10$ units) and increased serum concentrations of IgM was present. His serum cholesterol (213 mg/dl) and HDL (59 mg/dl) levels were raised with elevation in LDL (157 mg/dl).

Viral markers (HBsAg, Anti HCV), antinuclear antibody (ANA) and anti-smooth muscle antibody (ASMA) were negative. Urine analysis for copper, serum ceruloplasmin and alfa feto protein levels were normal.

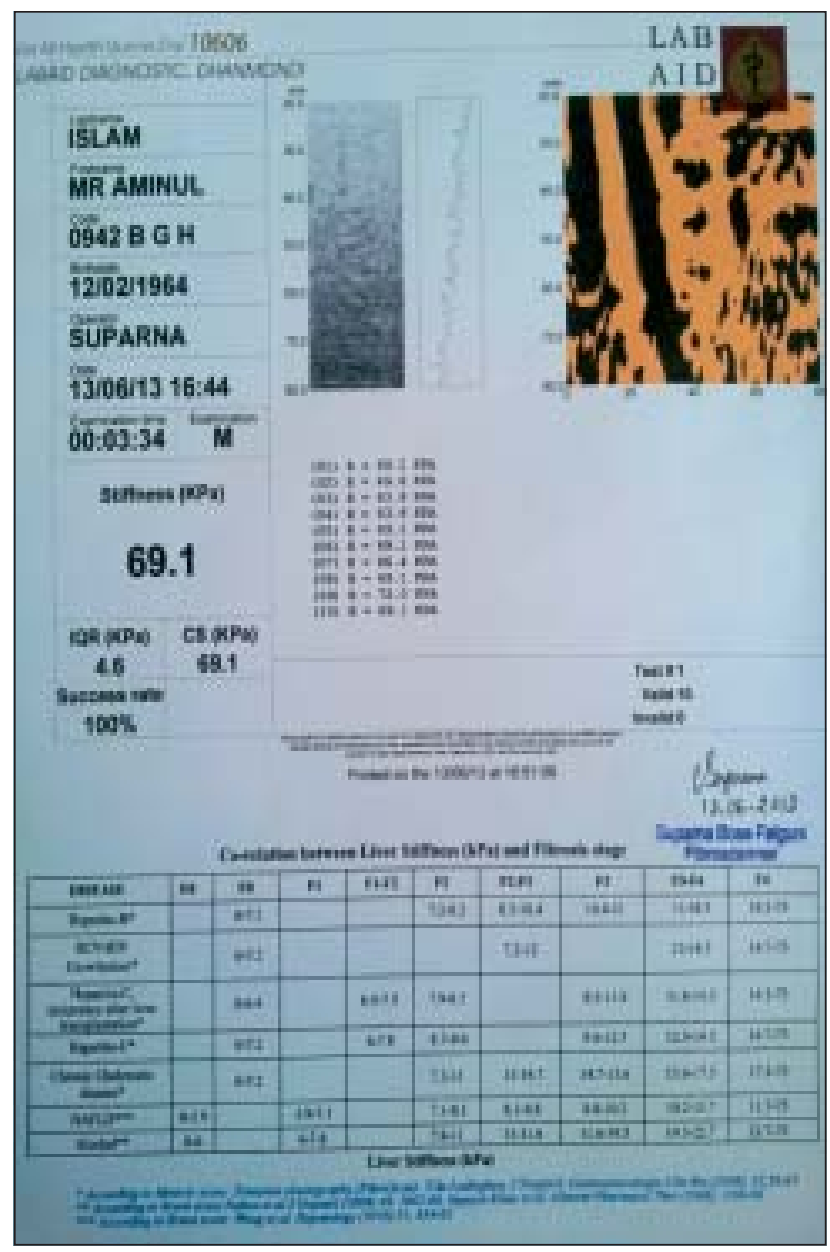

Fig.-3: Fibroscan showing severe liver stiffness.

So, the patient was finally diagnosed as a case of PBC with features of portal hypertension and Hashimoto's thyroiditis. Treatment was started with ursodeoxycholic acid (UDCA) $300 \mathrm{mg}$ twice daily and propanolol $40 \mathrm{mg}$ per day.

\section{Discussion}

Addison and Gull first described PBC in 1851. The label 'primary biliary cirrhosis' was adopted in 1949, even though not all patients were cirrhotic at diagnosis. ${ }^{4}$ In course of time, with clinical experience and research, a highly variable clinical course of PBC has been established.

The presentation of our patient was with fatigue followed by pruritus. Recent reports have found that, in newly diagnosed PBC patients, approximately one-half complain of fatigue and one-third pruritus. While many patients with PBC have preserved quality of life, fatigue may be a major factor that can impair quality of life. ${ }^{5}$ Extreme fatigue might also be associated with excessive daytime somnolence. ${ }^{6}$ Our patient complained of impaired quality of everyday life.

Approximately 25 to 50 percent of newly diagnosed patients have hyperpigmentation of skin. Jaundice is a later 
manifestation. Xanthomas from hyperlipidemia are also late manifestations that occur in less than 5 percent of patients. Xanthelasmas are more common and occur in approximately 10 percent of patients. ${ }^{7}$ Our patient lacked these features.

Hepatomegaly becomes more common as the disease progresses and is eventually found in approximately 70 percent of cases. The frequency with which splenomegaly is present on initial presentation has not been well described. Splenomegaly becomes more common as PBC progresses and is a sign of portal hypertension. ${ }^{8}$ Our patient had hepatosplenomegaly and features of portal hypertension.

The serum alkaline phosphatase concentration is almost always elevated in PBC, often to striking levels and is of hepatic origin. The value tends to reach a plateau early in the course of the disease and then usually fluctuates within 20 percent of this value. The serum levels of aminotransferases may be normal or slightly elevated, rarely increased more than fivefold above normal. They tend to fluctuate within a relatively narrow range and are of no prognostic importance unless they are 10 times normal or higher. The serum bilirubin concentration is usually normal early in the course of the disease but becomes elevated in most patients as the disease progresses. An elevated serum bilirubin is a poor prognostic sign. ${ }^{8}$ In this case elevated liver enzymes and normal serum bilirubin were present.

Increased numbers of eosinophils have been demonstrated in the blood and liver of patients with PBC, particularly in its early stages, suggesting that they may have a pathogenic role. ${ }^{9}$ Our patient had eosinophilia and he had no history of allergies.

AMA are the serologic hallmark of PBC. They are present in about 95 percent of patients with PBC. Most assays are 95 percent sensitive and 98 percent specific for PBC. ${ }^{10}$

The major debate in our case was that although this patient had a cholestatic picture and portal hypertension, his USG and MRCP showed normal hepatic parenchymal and biliary channels. AMA was positive and fibroscan confirmed liver stiffness.

ANA are found in up to 70 percent of patients with PBC. ${ }^{11}$ This case falls under the $30 \%$ PBC cases that do not have a positive ANA. Undoubtedly the combination of AMA positive and ANA negative in this patient had rendered the case more interesting.

One study including 29 asymptomatic patients with positive AMA and normal liver function suggested that the presence of AMA alone was a predictor of the eventual development of PBC. Liver biopsy revealed mild changes that were non diagnostic but consistent with very early PBC in 24 of 29 patients. Over the next 10 years most patients developed symptoms of PBC, laboratory signs of cholestasis and histpothological progression. ${ }^{12}$ As the AASLD criteria were met and other causes of cholestatic hepatic impairement were excluded, we did not opt for a liver biopsy in our case.

Lipids may be strikingly elevated in PBC. Serum cholesterol levels are elevated in at least 50 percent of patients. Patients with early PBC have mild elevations of low-density and verylow-density lipoproteins (LDL and VLDL) and striking elevations of high-density lipoproteins (HDL). This may explain why patients with $\mathrm{PBC}$, despite striking hypercholesterolemia, are not at increased risk of death from atherosclerosis. ${ }^{13}$ Our case showed similar results.

Other biochemical abnormalities in PBC include increased serum concentrations of IgM, ceruloplasmin and bile acids. ${ }^{14}$ This case had elevated IgM concentrations but a normal ceruloplasmin.

In an older report of extrahepatic autoimmune diseases (autoimmune thyroid disorders, sicca syndrome, systemic sclerosis, celiac disease) among patients with PBC, 84\% and $41 \%$ of the patients had at least one and more than two associated autoimmune disease respectively, in addition to PBC. Sjögren syndrome, including sicca syndrome was the most commonly associated autoimmune disease, seen in 66\% of the patients. ${ }^{14}$ Our patient had Hashimoto's thyroiditis.

An association between PBC and cigarette smoking has been suggested in some epidemiologic studies hinting that it is associated with more advanced fibrosis stage. In one of the studies, never-smokers were significantly less likely to have advanced fibrosis (METAVIR fibrosis score of 3 or 4) than patients who had smoked in the past or were current smokers (16 versus 33 percent). For each pack-year increase in smoking, there was a five percent increase in the likelihood of advanced fibrosis. ${ }^{15}$ It is our assumption that the development of cirrhosis in this patient's liver, being more evident on fibroscan might be due to his prolonged and current smoking habit.

So far as conservative therapy includes UDCA for improvement in liver function, cholestyramin resin and rifampicin for pruritus. Studies of patients treated with UDCA have suggested a good prognosis in patients initially diagnosed with mild disease who achieve a biochemical response to UDCA. Plasmapheresis is suggested in severe cases. Liver transplantation shows a 30\% recurrence in transplants. ${ }^{16}$ Our patient's pruritus improved with UDCA.

The prognosis of PBC has improved markedly during the past two decades. Many patients with early-stage PBC may 
have a normal life expectancy with treatment. Further genetic research may provide a bridge to clinical research, perhaps indicating predictive markers of disease progression as well as facilitating the basis for developing novel approaches to therapy by throwing light on the pathogenesis of PBC. Only these may determine why standard treatments for other autoimmune diseases are ineffective in PBC. It is hoped that a genetic explanation for the different patterns of disease presentation in PBC might be revealed and better targets for therapy still to be identified.

\section{Conclusion}

As PBC is uncommon in male, there should be a high degree of suspicion in an appropriate clinical scenario and/or biochemical cholestatic reports.

\section{Conflict of Interest: None}

\section{References}

1. Kaplan MM. Primary biliary cirrhosis. N Engl J Med. 1996; 335:1570-80.

2. Lindor KD, Gershwin ME, Poupon R, Kaplan M, Bergasa NV, Heathcote EJ. Primary biliary cirrhosis. Hepatology. 2009;50:291-308.

3. Angulo P, Lindor KD. Primary biliary cirrhosis. In: Feldman M, Friedman LS, Brandt LJ, editors. Slesinger and Fordtran's Gastrointestinal and Liver disease. 9th ed. Philadelphia (USA): Elsevier; 2010. p. 1477.

4. Addison T, Gull W. On a certain affection of the skinvitiligoidea-á-plana â-tuberosa. Guys Hosp Rept. 1851; 7:265-77.

5. Selmi C, Gershwin ME, Lindor KD, Worman HJ, Gold EB, Watnik $\mathrm{M}$ et al. Quality of life and everyday activities in patients with primary biliary cirrhosis. Hepatology. 2007; 46:1836-43.

6. Newton JL, Gibson GJ, Tomlinson M,Wilton K, Jones D. Fatigue in primary biliary cirrhosis is associated with excessive daytime somnolence. Hepatology. 2006;44:91-98.
7. Koulentaki M, Ioannidou D, Stefanidou M, Maraki S, Drigiannakis I, Dimoulios P, et al. Dermatological manifestations in primary biliary cirrhosis patients: a case control study. Am J Gastroenterol. 2006;101:541-46.

8. Dickson ER, Grambsch PM, Fleming TR, Fisher LD, Langworthy A. Prognosis in primary biliary cirrhosis: model for decision making. Hepatology. 1989;10:1-7.

9. Yamazaki K, Nakadate I, Suzuki K, Sato S, Masuda T. Eosinophilia in primary biliary cirrhosis. Am J Gastroenterol. 1996;91:516-22.

10. Muratori L, Granito A, Muratori P, Pappas G, Bianchi . Antimitochondrial antibodies and other antibodies in primary biliary cirrhosis: diagnostic and prognostic value. Clin Liver Dis. 2008;12:261-76.

11. Wesierska-Gadek J, Penner E, Battezzati PM, Selmi C, Zuin M, Hitchman E,et al. Correlation of initial autoantibody profile and clinical outcome in primary biliary cirrhosis. Hepatology. 2006;43:1135-44.

12. Metcalf JV, Mitchison HC, Palmer JM, Jones DE, Bassendine MF, James OFW. Natural history of early primary biliary cirrhosis. Lancet. 1996;348:1399-402.

13. Sorokin A, Brown JL, Thompson PD. Primary biliary cirrhosis, hyperlipidemia, and atherosclerotic risk: a systematic review. Atherosclerosis. 2007;194:293-99.

14. Culp KS, Fleming CR, Duffy J, Baldus WP, Dickson ER. Autoimmune associations in primary biliary cirrhosis. Mayo Clin Proc. 1982;57:365-70.

15. Corpechot C, Gaouar F, Chrétien Y, Johanet $C$, Chazouilleres O, Poupon R. Smoking as an independent risk factor of liver fibrosis in primary biliary cirrhosis. J Hepatol. 2012;56: 218-24.

16. Joshi S, Cauch-Dudek K, Wanless IR, Lindor KD, Jorgensen $\mathrm{R}$, Batts K, et al. Primary biliary cirrhosis with additional features of autoimmune hepatitis: response to therapy with ursodeoxycholic acid. Hepatology. 2002;35:409-13. 